\section{Neueste Einblicke in die Laserzahnheilkunde}

Dentale Diodenlaser überzeugen durch eine meist narbenfreie Wundheilung und eine verbesserte Keimreduktion. Durch die Entwicklung des SIROLaser Blue stehen dem Anwender inzwischen 3 Dioden - Blau, Rot und Infrarot - zur Verfügung, mit denen mehr als 20 Indikationen behandelt werden können.

Speziell die blaue Wellenlänge bietet mit ihren $445 \mathrm{~nm}$ einige Behandlungsvorteile wie das Arbeiten im Non-Kontakt-Modus bei chirurgischen Eingriffen. Welche Erfahrungen Anwender bisher mit der blauen Diode gemacht haben und wie die Wel- lenlänge in Zukunft noch gezielter eingesetzt werden kann, wurde den Teilnehmern der Dentsply Sirona Laser Days umfangreich dargestellt. Insgesamt 52 Experten aus 21 Ländern kamen vom 29. September bis 1. Oktober im Hotel Bachmair am Tegernsee zusammen, um sich in zahlreichen Fachvorträgen und Workshops über die vielfältigen Behandlungsmöglichkeiten von Dentallasern auszutauschen. Dabei profitierten die Anwesenden auch vom persönlichen Kontakt miteinander: Insbesondere das Abendessen im bayrischen Ambiente am 2. Tag und die insgesamt lockere Atmosphäre des Events ermöglichten es, Kontakte zu knüpfen und so die internationale Gemeinschaft in der Laserzahnheilkunde weiter zu stärken.

„Unser Ziel ist es, allen Interessenten einen möglichst umfangreichen Einblick in die neuesten Forschungsstandpunkte im Bereich Laserzahnheilkunde zu gewähren. Deshalb haben wir die Teilnehmer freitags eingeladen, am 25. Jahreskongress der deutschen Gesellschaft für Laserzahnheilkunde teilzunehmen“, erklärt Jan Sie- 


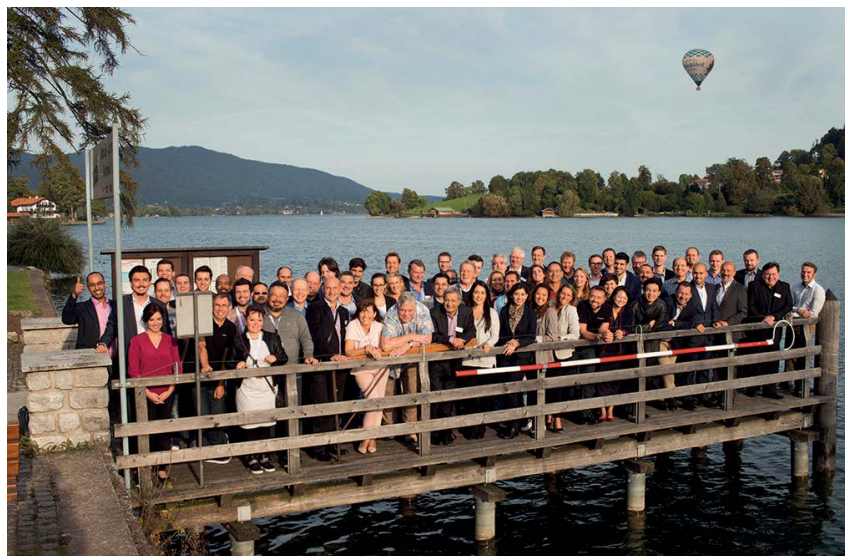

nutzte seinen Workshop, um die Teilnehmer an den praktischen Einsatz der multiplen Wellenlängen des SIROLaser Blue heranzuführen. Neben den klinischen Indikationen ging er dabei unter anderem auf die Laseranwendung zur Vorbereitung der Abdrucknahme mit CEREC ein.

fert, Group Vice President Instruments bei Dentsply Sirona. Unter dem Motto „Von den Ursprüngen zu neuen Horizonten“ referierten hier zahlreiche renommierte Experten aus aller Welt. Dr. Carlo Francesco Sambri
Auch am Samstag wurde den Teilnehmern der Laser Days ein umfangreiches Veranstaltungsprogramm mit klinischem Schwerpunkt geboten - im Vordergrund stand dabei die Verwendung der blauen
Diode mit 445nm in der Praxis. Ein Workshop, in dem die Teilnehmer über die $\mathrm{Zu}-$ kunft der Laserzahnheilkunde diskutieren konnten, rundete das Programm der 3. Internationalen Dentsply Sirona Laser Days ab.

Auch in Zukunft können sich interessierte Zahnärzte in der Dentsply Sirona Laser Plattform über aktuelle Trends der Laserzahnheilkunde informieren. Zusätzlich bietet das integrierte Laser Forum reichlich Raum für den direkten Austausch mit anderen Laser-affinen Kollegen: http:// www.sirona.com/de/sirolaser

Aus einer Pressemitteilung der Dentsply Sirona,

A-Wals bei Salzburg 